\title{
Forming and Development of Performing Skills with the Blind Accordion Players
}

\author{
Igor Syroyezhkin \\ Russian State Specialized Arts Academy \\ Moscow, Russia
}

\begin{abstract}
The article is dedicated to the problem of the methodology of the accordion playing of the blind students. The author declares that the right work of the hands is an intellectual process, so it may be taught to a blind student.
\end{abstract}

Keywords-music education; blind students; methodology; accordion playing; performers equipment

\section{INTRODUCTION}

For the blind musicians the issues connected to the performers skills development are the priority. It is characteristic with the eyesight disability people to be reserved, afraid of the outside world and muscle constraint, movement restriction and as a result stiffness of performing equipment. Many people start studying music seriously at an adult age, more often at a specialized college, having only episodic classes in the boarding schools. So that's why when they get to a college or an institute they often don't have an idea of the right sitting, the position of a music instrument, performers equipment liberty, about the work in the slight finger technique and about the physiology of the movement process. Lack of knowledge and understanding of such issues leads to the slower movement of the technical process of a musician and in worst case to the professional diseases.

\section{MUSClE FREEDOM AND HAND LIBERATION}

Sensation of freedom is the first priority of a musician's formation. Lack of a clear understanding of muscle freedom is the main factor, which creates favorable ground for different defects, which later appear with the movement equipment. No hope for a more or less regular hand work is possible without this sensation.

A human hand is a unique perfect apparatus, created by nature. Its abilities, multiple functions, reaction and brain orders fulfillment ability has nothing equal in the world. Hands have the maximum connection to brain. Everything that has been created by a human in different areas could not be created without a hand. It is impossible to learn this perfect apparatus without knowing it.

But the accordion players sometimes treat their hands as consumers, making them work with no rest, without thinking about the conditions hands work. They often look for the reasons of their hand imperfection and professional failures in a wrong place. An accordion player who does not know his own hands can not give his students the right knowledge in this issue.

It is necessary to stress an importance of the preliminary learning of muscle freedom and an ability to move the muscles in the elementary hand movements before getting to work with a music instrument. In a particular way every human is familiar with a sensation of muscle and joint freedom as it sometimes appears in our everyday life. Still the subconscious muscle liberation does not remain the human memory that's why we can't use it voluntary.

Hands muscles are usually free, for instance when walking or when they hang loosely along the body, since the body itself puts them into this position. If we try to pay our attention to feeling all the specifics of this condition and try to memorize it firmly, then, after some training, we'll be able to use it in hand movement during some work fulfillment.

Hands falling free down the body is the best position, which allows to relax all the muscles, since there are no supporting efforts. Muscle relaxation confirmation is a sensation of heaviness, completeness, space and comfort inside the hands. Then one should lightly push hanging hands forward by the muscle impulse and observe as they swindle until they fully stop. In such free hand swindling the sensation of hand weight won't be the same as it changes in some slight doses and most of the time these changes can be seen in a hand itself: when changing the direction of the movement a hand may feel almost weightless and on coming to the body the filling of the hand will be more felt.

It is useful to compare an immovable hand sensation with the swinging one. This sensation difference should also be caught and memorized. We should stress that one should find a balance in hand observation, as well as the similarity of different sensations and to estimate it from the position of "better", "worse", "more comfortable", "less comfortable". For definition of all the verified specifics it is necessary to find words, which reflect this or that sensation. In this case sensations are learnt better and are repeated easier during exercise, since a word helps to an emotional-will effort.

All the moments when a sensation of comfort will appear in hands should be remembered and developed, since it is a particular comfort and naturalism feeling that reports to the central nerve system about the regular muscle functioning. When the general feeling of freedom is learnt, one should observe the condition of the upper part of the 
hand: an arm, a shoulder, a hand, every finger. All conditions, learnt by the hand in general, are necessary to feel in every part of the hand in the same positions - immovable and swindling. It is impossible to miss any part of a hand: everything should be thoughtfully studied. Then these sensations should be checked in a relaxed and a taut body positions.

\section{PASSIVE AND ACTIVE LIBERTY}

A person's mood and his posture are also connected. A natural, comfortable, convenient posture is one of well-being conditions. The right posture is not only the choice of position, but also the feeling of the tone of the body.

An idea of a "tone" is associated by the physiologists with an ancient level of reflection activity of the nervous system and the long keeping of a particular muscle activity. N.O. Bernstein believes that muscle tone is the muscle and nerve readiness for action. A relaxed and toned up position the same movements are felt differently because of the positive or negative muscle tone. In comparison one can easily observe that a relaxed position creates a passive muscle mood - hands become passive and the attention lowers. A toned posture creates active muscle mood - all movements become light, hands obey and the attention sharpens. An active position all the nervous-muscle apparatus aids the movements, while the passive position does not do it. The correct posture aids a better keeping of the body because of the back and chest muscle stretching. The stoop posture unequal muscle stretching lacks the hand and chest active body support, interior organs are pressed, which also does not provide for a better conditions of work.

Thus, there are two categories of muscle liberty - the passive and the active one. Passive muscle freedom is relaxation is characterized by the sensation of weight, heaviness and filling. Such muscle relaxation is necessary for rest: it allows the muscles to rest and to recuperate. But working with relaxed muscles leads you to the fatigue. Active muscle freedom is characterized by a sensation of lightness, shape, readiness to work. It is in this condition that the muscles should work. In order to get the muscles to such condition one needs a particular tone tension.

\section{NATURAL POSITION OF FINGERS}

The next phase is finger work observation. On a free falling hand one should feel comfort and naturalism, paying special attention to the condition of every finger in turns.

Having studied fingers condition on an immovable hand one should continue studying it folded and unfolded. A muscular effort of every finger should be minimal. Every movement should be felt and memorized. On turning of any finger one should control the fingers that are not involved. They should not be tense. The first and the second finger are rather independent, the third, the fourth and the fifth are connected, so when any of them is moving the other repeat its movements. This phenomenon should be considered regular and it is unnatural to try to leave any finger unmoved during the work of the others. It is particularly necessary to control the work of the fourth finger: it is the most «uneasy» finger. It demands cautiousness and special attention. It is less strong and movable than the third and the second fingers, but systematic development may improve its qualities.

The finger's natural position is the position of the switched off folding and unfolding. It is easier to bring the fingers to the freely relaxed hands, then every finger has its own particular place. An accordion player should feel the slightest disruption of the natural position and should be able to take the necessary measures for the immediate mistakes correction. The first and the fifth fingers are the most inclined to this natural disruption: in such cases they are usually either stretched or hooked. Natural position disruption should be considered phalanx sagging: it is dangerous by increasing of the pressure to the hand. It also should be marked that any finger can be released only in a natural position.

\section{WHOLE BODY CONTROL}

At the same time with liberation of hand muscles the same work should be provided for the general body muscles, since tensions, which appear during the instrument playing will significantly prevent movement accuracy and lower durability. Unnecessary tensions should be looked in the body, legs, face and neck muscles. Long tension in the face and neck may lead to headaches, since brain blood circulation is disrupted which leads to memory, eyesight, hearing limitation and fast fatigue.

All the above mentioned tasks provide the preliminary stage of hand liberation development. Hand muscles observation and the whole body is necessary to control in any work: in this case self-control and muscle tension regulation will become a habit and success will come in a shorter time period.

\section{JOINT PHYSICAL AND MENTAL EFFORTS}

The following perfection of the muscle-joint sensations should be combined with an instrument and without it. In a certain time a person may stop practicing without the instrument at all. Such exercises may be necessary only in a case when some sensations of some should be specified. In the right organization of the playing movements an important role is given to an exact imagination of an expected action. Such words as: heaviness, weight, comfort define the sensation of muscle freedom. Such words as lightness, comfort, naturalism also define the sensation of muscle freedom.

During the classes of accordion playing one should use the words, which accurately define the condition, which is necessary a particular moment for the finger and hand movement. For example, fingers may be light or active, hand movements are natural, calm, strong. Such word formulas will aid in hands quick preparation and a more precise movement organization.

Psychophysical laws of the working movements organization of a musician are common for everybody. The basis of the muscles work at any music instrument is an 
impulse principle. It considers that every sound extraction passes through three conditions stages, which change each other simultaneously.

Movement readiness represents muscles active liberty, when fingers are in natural position, an economic effort is applied, which is necessary only for a particular position. Sound extraction. Every music instrument has its own specifics, that's why there are a lot of touches ways. The choice of a movement for the sound extraction is defined by the sound tasks. The trick is to make a short effort, about an instant of a second, which is enough to hear a sound. After the hearing of the sound an effort should be eliminated. For example, at the accordion playing there should remain only an effort, meant for a light key touch. There should not be a single deep touch, since it is the deep touch that harms. It is the deep sound that leads to the hand diseases.

There are two types of the muscle activity: dynamic and static. Dynamic activity is meant for the transition of the body or its parts in the space and is connected to movement. The static is meant for keeping of the body or its parts in a stable position. During dynamic activity oxygen accumulation increases lightly depending on the muscle power lifting. The static increases the oxygen accumulation in comparison to the relaxed state. Blood circulation decreases as the constantly strained muscles press on the blood vessels. Metabolism also decreases since intoxicated substances are collected in muscles, as the result tumors appear, which are located on hands and palms. Blood circulation and metabolism disruption leads to the organic changes in muscles and bones. So, touching the keys deeply is a great enemy of instrumentalism.

All three stages of muscle conditions - preparation, sound extraction, keeping of the sound are necessary to be controlled and realize every stage. Slow movements contribute to a brighter detail realization, better memorization of all the specifics of muscular work and a better correction of the muscular tension. It is necessary to understand that hand work is first of all an intellectual process. In slow movements it is necessary to control every detail of the movement, then, as they become more trained, it is necessary to control the hands ' general condition and then to fasten or to automatize the movement. From time to time it is necessary to come back to the slow rhythm to prevent possible mistakes.

It is necessary to specify the finger work in the fine technique. The major principle of their work is free falling. Niccolo Paganini's fantastic hand technique resulted from the knowledge and following of the natural finger falling. Paganini's objective was to avoid tension at all and to justify "natural finger falling" application. So, what is the essence of the "free finger falling"? Such finger movement we often use in our everyday life. But the difficulty is that we do it unconsciously. Now it should be realized again. In order to understand and to feel the essence of this movement some exercises should be made.

Sit down at the table, put an elbow on it, raise your arm and a hand with free fingers, take away all extra muscular power, control your fingers - they should fall freely down to the position where they should not be kept. The most important thing is to feel the natural free finger falling. Their internal condition in the moment of the fall should be very comfortable. But this is just a scheme. No work at this condition can be done. It is a passive falling.

Having practiced the technique of free finger falling it is necessary to practice active falling. Here a muscle impulse is added, when muscles get an extra energy, necessary for sound extraction. The difference of the active falling from the passive one is that fingers should start their fall lightly, quietly and then move up freely, keeping its naturalism and comfort, muscular impulse, which muscles got at the beginning of the fall should not provide tension, it is switched on only during the moment of sound extraction. Uninvolved fingers may try the following movements (esp. $3,4,5$ ), but they should not be tense. For a more complete and profound free passive and active finger falling one should use a stage learning of all the elements. Using this principle at the instrument before finger falling a light flight of the finger is necessary for a better work movement activation.

A great role in the correct movement skills formation is played by the attention. It is closely connected with the psychic processes. Ushinsky used to say that attention is that our soul's only door, through which everything that we have in our conscious comes. An attentive person having all of his brain centers activated hinders the others: a person releases himself from unnecessary nervous strain and it results in the muscular relaxation of those organs, which are not involved.

When doing different movements in the period of their mastery one should attentively follow the change of the muscular sensations, compare them and to choose the best and to fix them. Analysis and comparison of the feelings allows to get a better clue to their understanding. Here selfobservation plays an important role.

Two-hands work is controlled in three ways: by touch, by eye and by ear. During the initial learning of the movements hand work is controlled by touch and by eye. It is necessary to learn to feel deeper what happens inside the hands: finger posture, joints, hands in general. Eyesight controls the external side of actions: finger position, joints, hands in general. By the balancing of internal and external sides of movement sound result should be taken in consideration in order to realize and stabilize the connection of the movement to the sound. When learning the movements and getting the automatism the control gets to the ear.

\section{PARTICULARITIES OF PlAYING THE ACCORDION}

Some specifics of sound extraction and the vertical position of the keys on the accordion is necessary to be explained. An important problem is working out of the correct contact of the fingers and the key-cords. Posture and development of the performers' skills of the accordionplayer depends on it. Fingers are a part of a performing apparatus, which is closely connected to the keys. According to H. Neihaus here is "a front line", which should prepare and protect the "rear part" (a hand, an arm, a shoulder, a body). A great attention should be given to a principle of the 
natural weigh of the hand. Applied to the accordion players is will not vary from the general piano principle. Unlike the pianists, whose hands and body find a perfect posture on the keyboard, the accordionists have no such posture, since their instrument keys are positioned vertically. In order to avoid extreme muscles tension, the accordionists still have to find relaxation of the keys.

A hand's weight during its movement along the keys helps the movement and when moving up it becomes a brake. The power of weight is overcome by a soft and constant movement of the hand up. Here the muscles of the shoulders take part together with the hand and fingers. Lack of understanding of this process leads to lack of movement in shoulder, hand and fingers.

As a result of the specifics of sound extraction on the accordion, the sound character does not always correspond to the hand movements. For example, a sound may be very mild and weak at a tense hand. Such tensions are difficult to be found, a constant control of the sound quality is needed and the minimal efforts for getting the right sound. The nature of the accordion sound extraction allows all the performers to get a complete, full sounding with light pressure finger tension. At the same time the right hand tension has the minimum of tension and the left has only the fingers and the hand tense. This tension minimum is necessary for fixation of the position on the keys, since in the opposite case the hands will loose its contact and will lead to the superficial playing. Some accordionists at the highly dynamic levels of sounding psychologically are aimed at an extreme hand and shoulder tension, thus making their movements heavier. It is necessary to get the right sounding with the minimum conditions. The sound power depends on the physical insensitivity of the efforts only for those parts of the hand, which provides the movement of the skin.

\section{FreEDOM SENSATION IS A MUST}

There's a famous and an undoubted fact that a student who gets the maximum result is the one whose movement apparatus is free. Sometimes it happens that the more an accordion player studies, the worse it plays. Everything is explained by the fact that all the forces of such players are given to overcoming of their obstacles and not to the artistic performance. The more they fight the obstacles, the more they have. It is especially obvious on stage: it is difficult for a musician to concentrate on the artistic side of their performance since they spend a lot of nervous and physical forces to overcome technical difficulties. Such difficulties increase lack of confidence, arise the sense of fear, nervousness and fussiness. Fixing their attention on the process of overcoming of the difficulties they disturb artistic job even more. And if in these cases a nervous breakdown happens, then uncertainty and fear increase, since their memory fixes the previous failures. One of the main reasons of such situations is an unconscious hand movement.

The stage environment makes the brain work full power, there are extreme conditions, so a performer makes mistakes, stops and breaks down. It is especially typical for those colleges where students have to perform an unfinished work due to the lack of training. Such performances leave a negative trace in the memory and increase the sense of the stage fear. A great importance of the positive stage feeling have the positive emotions. Combination of positive emotions provide the feelings of self confidence, inspiration and provide the development of the creativity of the musician. Negative emotions don't allow rationally and effectively to use your abilities and talents.

A human has a protective system which is aimed at protecting the body of negative and dangerous environment influences. This protective system has a lot of ways to signal the brain about different changes in the organs, muscle tissues, etc. A sensation of uneasiness and fatigue are the first signal of hands ill being. Fatigue is one of the body's natural expressions. If we don't pay attention to it and keep studying, then it will become even more acute. For some time the body's endurance will be enough, but slowly it will attack the other parts of the body. In this case it will involve the neighboring muscles and as a result muscular energy will decrease and quickness and accuracy of the movements will decrease. It will lead to the fact that even in simple movements almost all muscular system will be involved. It will decrease the hand work and will create negative conditions of work for the whole body. It will lead to pain that will cover all the body.

On different reasons musicians sometimes have to play the instrument in spite of the pain. Long pain feeling may lead in the end to the serious organic changes in the muscles and bones, to constrictions and the general body disruption. Ignoring of the signals may be fatal and sometimes the musicians pay a great price for their inattention.

\section{CONCLUSION}

At the music instrument playing a lack of the knowledge how to spread the muscular efforts results in very fast problems in the body. The most typical reason of appearance of the disruptions in the accordion playing is the extreme effort in pressing the keys. Rational spreading of the efforts and keeping the key on the accordion is an ability to press the keys deeply and to keep them with one effort. Both hands fingers should always be light, sensitive and active.

The accordionists sometimes have a habit to raise the right or the left elbow, or even both ones. Such elbow position is necessary only in the case when a special beneficial position should be given to the fingers. If there's no such a necessity, the elbow should be kept down. In this way we avoid extra power for the elbow shoulder and the body. In the opposite case a habit to play with constant muscles constraint will appear. It will lead to the lack of endurance and loss of technique. It is necessary to give a way to the muscle relaxation. Of three stages of the hand movement for sound extraction-preparation, sound extraction, key pressing, there will be only one working stage - sound extraction, the other two will be hand relaxation.

Relaxation moments for the muscles also may be considered the pauses, censure, movement of the hands to the other objects. Disruptions in the work of the movement 
apparatus appear as the result lack of movement school, ignoring of psychophysical laws of the muscle work. All this leads to some disruptions at which sound and health and technique may suffer. The right teaching of the movement skills of the musicians ' hands excludes such problems. But it requires great patience and will power. Still we can't get to professional summits without it. 